\title{
RFID Anti-Collision Detection Algorithm Based on Improved Adaptive N-Tree
}

\author{
https://doi.org/10.3991/ijoe.v14i10.9310 \\ Yuxia Li $\left({ }^{\bowtie}\right)$ \\ Jiangsu Maritime Technical Institute, Nanjing, China \\ 64387892 @qq. com \\ Zhinan Zhou \\ Hebei Agricultural University, Baoding, China
}

\begin{abstract}
This paper proposes a type of improved adaptive N-tree anticollision algorithm based on the traditional one for RFID system by combination with maximum likelihood estimation and probe pre-detection. This algorithm inherits some features from Alpha- and tree-based anti-collision algorithms and effectively restrain the star-vation of the two algorithms. It has also filled in the gaps of tag collision with higher probability. The study turns out that the improved adaptive $\mathrm{N}$-tree anti-collision algorithm as proposed can feature adaptive choice of the value $\mathrm{N}$ of the tree, length breaks of free timeslots, restraints on defects such as more tag classification and higher collision probability just as what the traditional tree-based algorithm has. N-tree built by levelto-level frame identification eliminates the free timeslots, and improves the tag identification precision for the RFID system. The results from simulation experiment reveal that the algorithm proposed in this paper has lower Error Sampling Reckon (ESR) and Throughput Rate Deviation (TRD), and features large throughput rate $(87 \%)$, low delay of tag recognition and minimum timeslots, and etc. hence to be better applied in large-scale logistics and other fields where fast information recognition is involved.
\end{abstract}

Keywords - radio frequency identification, anti-collision algorithm, throughput rate, identification time, free timeslot

\section{Introduction}

Radio Frequency Identification (RFID) is an untouched detection and recognition technology, which access to a certain range of target information by way of the scanning of radio frequency signals that is completely controlled by computer. It features high efficiency, easy to use and high adaptability to various harsh environments. Today, this technology has been widely used in many transportation and logistics systems such as Internet of Things, shipping, port, land transportations [1-3].

RFID system consists of backend server, RFID reader and RFID tag. A single RFID reader gives a command for query, to which a number of tags respond within the scope of it. The messages responded by multiple tags will collide with each other 
when they return to the reader, thus resulting in the information aliasing [4-6]. It is therefore significant for people to study anti-collision approaches of tag information or to differentiate the collided information in practices.

Currently, the anti-collision algorithms of RFID system mainly include: (1) Alohabased anti-collision algorithm which suppresses information collision by randomly extending the start time of information transmission [7-9] (2) Tree-based anticollision algorithms which subdivides the tags where the information collisions occur into a number of subsets for unique identification [10-14] (3) A Aloha- and tree-based superposition hybrid algorithm [15-21]; The above three algorithms all have limitations in different degrees.

A type of improved adaptive N-tree anti-collision algorithm is built based on the traditional one for RFID system, and with the incorporation of maximum likelihood estimation and probe pre-detection. This algorithm inherits some features from Alohaand tree-based anti-collision algorithms, and more importantly, it fills in the gaps that the two algorithms fail to actively suppress the starvation and allow higher probability of tag collision. This paper provides a new idea for scholars to study the subject about the RFID system anti-collision.

\section{RFID system model and CT algorithm}

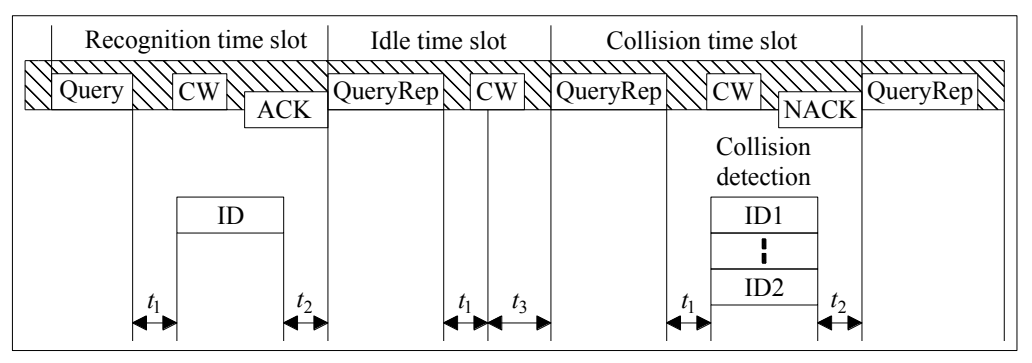

Fig. 1. Transfer model of RFID system

As shown in Fig. 1, a model is built for mutual information transmission between reader and tag in an RFID system [22]. The model includes identification, free and information collision areas. In the identification area, an RFID reader issues the Query command, $t_{1}$ and $t_{2}$ respectively represents the time when the tag accepts the command and make a response and the time when the information is transferred to the reader; $t_{3}$ is the time when the reader does not receive the response after sending the command. At this time the system judges as free area; ACK and NACK are respectively identifiable timeslots and collision timeslots issued by reader.

The principle of information collision detection in CT algorithm is shown in Fig. 2. $\mathrm{CT}$ algorithm mainly uses the ME code to track the tag information. ID numbers "0" and "1" represent the positive and negative migration of the voltage, respectively. The positive and negative voltages in the positions where the information of different tags collide are offset each other. Subcarrier signal received by reader is an integer Bit, by which the collision can be located [23]. 


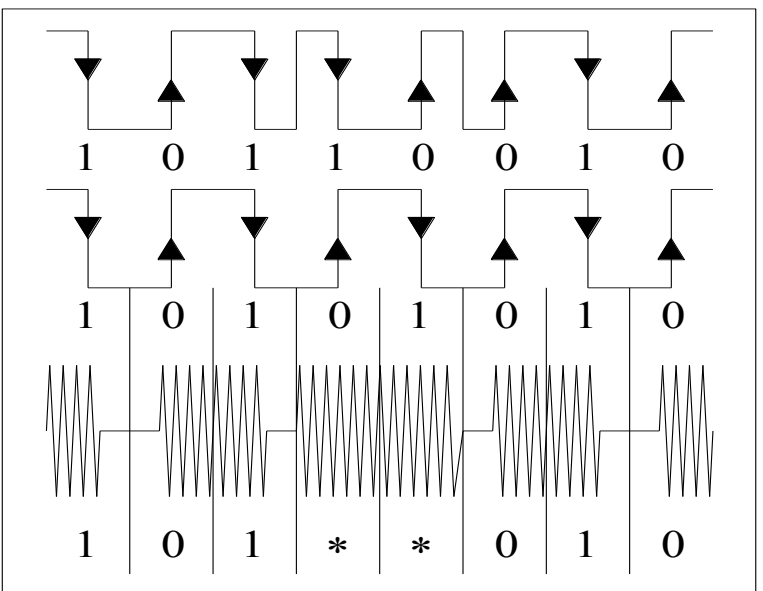

Fig. 2. Principle of anti-collision detection in $\mathrm{CT}$ algorithm

\section{Designing the improved adaptive $\mathrm{N}$-tree anti-collision algorithm}

\subsection{Computation process}

An improved adaptive N-tree anti-collision algorithm is constructed by incorporating maximum likelihood estimation and probe pre-detection. This algorithm adaptively chooses the $\mathrm{N}$ value of the tree and reduces the length of the free timeslot [24,25]. Suppose the number of tags is $\mathrm{n}$, the frame length is $\mathrm{N}$, the timeslot is $\mathrm{r}$. The probability $\mathrm{p}$ that there are $\mathrm{w}$ tags in the timeslot $r$ is

$$
P(w, r, n, N)=\frac{(-1)^{r} N ! n !}{r ! N^{n}} \sum_{j=r}^{\min (N,\lceil n / w])}(-1)^{j} \frac{(N-j)^{n-j w}}{(j-r) !(N-j) !(n-j w) !(w)^{j}}
$$

According to the Eq. 1, we can obtain the probability that no tag responds to the request issued by the reader in e timeslots as follows.

$$
P(e, n, N)=\frac{(-1)^{e} N ! n !}{e ! N^{n}} \sum_{j=e}^{N}(-1)^{j} \frac{(N-j)^{n}}{(j-e) !(N-j) !(n) !}
$$

The response of the tags in a scope of single reader belongs to an independent event, which fits well to the maximum likelihood estimation function. When $N, w, e$ are known, the number $n$ of tags can be estimated as

$$
\widehat{n}=\arg \max \left(P(e, n, N) \mid N=2^{Q}, n \geq N-e+c\right)
$$




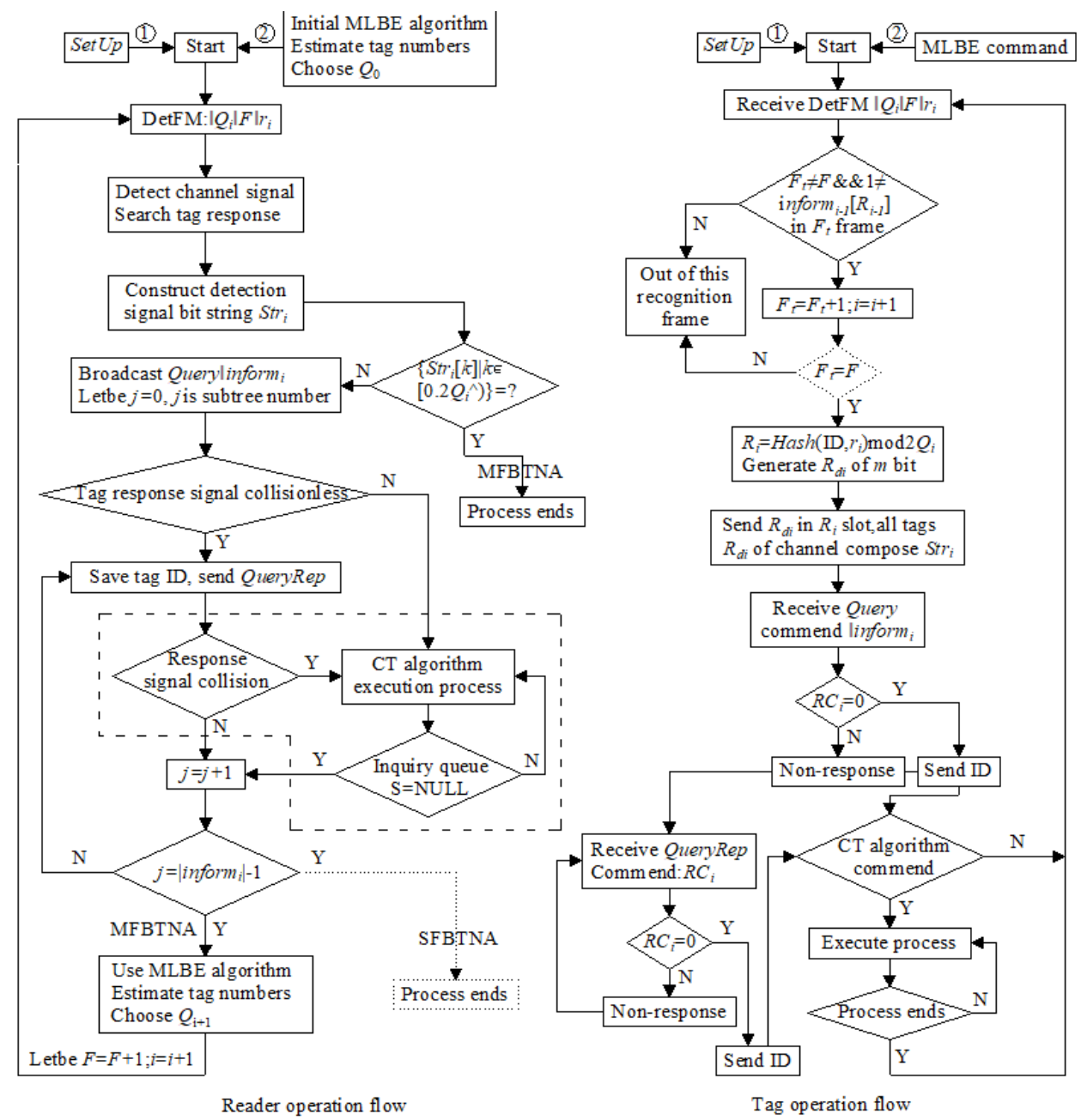

Fig. 3. Flow chart of an improved adaptive N-tree anti-collision algorithm

According to Eqs. 1 and 3, the adaptive N-tree anti-collision algorithm constructed in this paper is shown in Fig.3. Specific procedures are given as follows:

(1) Initialize the relevant parameters of the maximum likelihood estimation, the reader issues a Query command, perform the initialization after the tag in the scope of reader receives the command, and calculate the value $\mathrm{R}$ :

$$
R=\operatorname{Hash}(I D, r) \bmod 2^{Q}
$$

Suppose the response of the timeslot $\mathrm{R}$ is $R_{b}$. The reader detects the response sent by each tag. 
(2) Detect whether there is a collision during the tag response, if this is the case, save the tag ID, and the reader issues the QueryRep command at this time and judge the collision situation by the tag response; if not, execute CT algorithm.

(3) Determine whether the tag is recognized, and the number of tags is determined by maximum likelihood estimation.

(4) Detect the probe bit string $\operatorname{Str}_{i}$ according to the probe pre-detection.

(5) Confirm whether CT algorithm is activated according to the collision situation, save the tag ID if there is no collision in the whole process. It is proved that the tag is not lost and the first set of sub-trees are formed. Repeat the process until the classification of all tags is completed, and the entire process is concluded.

\subsection{Analysis of algorithm performance}

The initial value $\mathrm{Q}$ and optimal value $\mathrm{Q}$ interval and the probe length $\mathrm{m}$ in the maximum likelihood estimation algorithm are deduced to make its throughput rates achieve the best.

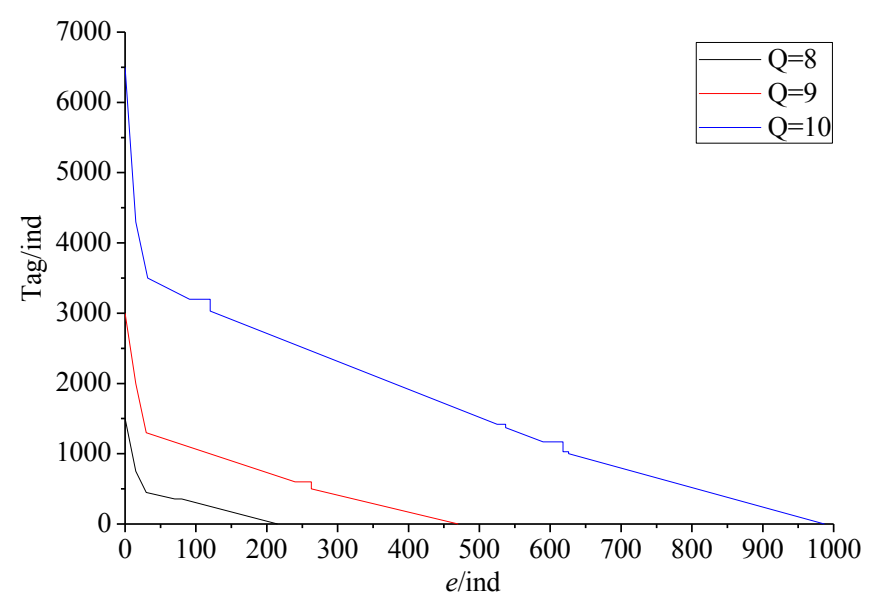

Fig. 4. Curve of label estimation as a function of variable $e$

When $\mathrm{Q}$ takes a fixed value $(\mathrm{Q}=8,9,10)$, the curve of the label estimations as a function of variable e is shown in Fig. 4. As can be seen from the figure, as the value $\mathrm{Q}$ increases, the number of labels in the system is also estimated to increase gradually; when $e=1$, the maximum estimated value of the labels can be available.

Suppose $P(k)$ is the probability that the label has no any response in any timeslot, then

$$
\left.P(k)\right|_{k=0}=\left(1-\frac{1}{2^{Q}}\right)^{n}
$$

$\mathrm{E}(\mathrm{k})$ is the expected value that value $e$ reaches the best 


$$
\left.E(k)\right|_{k=0}=\left.\sum_{k=0}^{2^{Q}-1} P(k)\right|_{k=0}=2^{Q}\left(1-\frac{1}{2^{Q}}\right)^{n}
$$

$\mathrm{e}>1$ and $\mathrm{E}(\mathrm{k})>1$, the number of labels is estimated, then

$$
2^{Q} e^{-n / 2^{Q}} \geq 1
$$

According to Eq. $7, \ln 2\left(2^{\mathrm{Q}} \mathrm{Q}\right)>\mathrm{n}$, suppose

$$
f(Q)=\ln 2\left(2^{Q} Q\right)
$$

Take a derivative with respect to Eq. 8 , then

$$
f^{\prime}(Q)=\ln 2\left(2^{Q}+2^{Q-1} Q^{2}\right)
$$

$f(\mathrm{Q})$ increases monotonically. Let $T_{M L B E}$ be the average time-consuming estimated from the number of tags, then

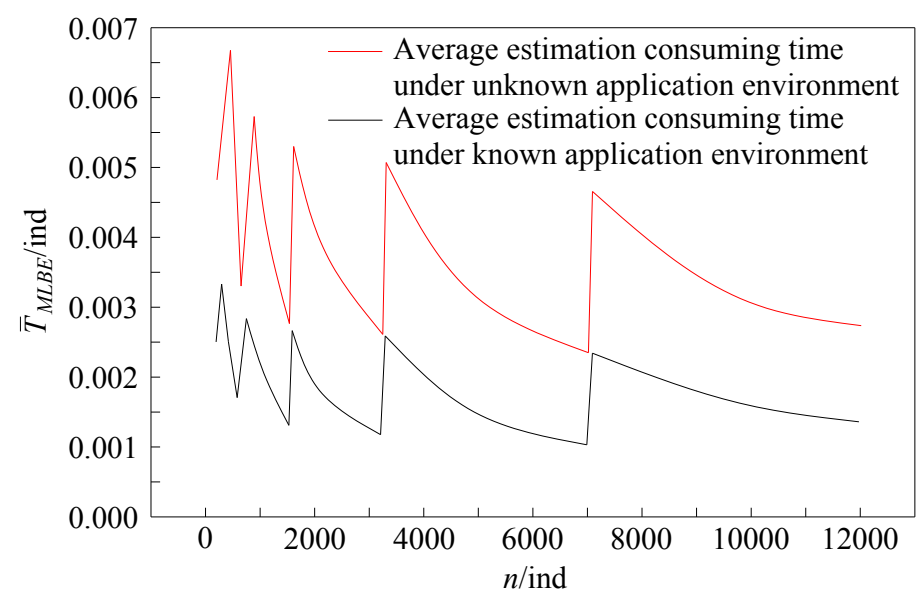

Fig. 5. Curve of average times consumed in the estimation of tags

$$
\bar{T}_{M L B E}=\frac{2^{1}+2^{2}+\cdots+2^{Q_{\min }}}{n \times D R}
$$

According to Eqs. 5 - 10, the average time spent in the calculations in the cases of the unknown labels and the approximate number of known labels is shown in Fig. 5. It can be seen from the figure that, if the number of tags is unknown, the estimation of the number of tags takes $0.0068 \mathrm{~ms}$ at most; $0.0034 \mathrm{~ms}$ when the approximate number of tags is known. 


\section{$4 \quad$ Verification of simulation experiment}

The algorithm proposed in this paper is compared to the traditional RFID anticollision algorithms such as OQTT, CT, FSA and SFBTNA to verify the availability of the proposed algorithm. The estimation error and the throughput error are two important indicators used to evaluate the estimation and throughput precisions of the algorithm. The estimation error (ESR) is calculated by

$$
E S R=\left|n_{i}-\hat{n}_{i}\right| / n_{i}
$$

The throughput error (TRD) is calculated by

$$
T R D=\left|\frac{\left.E\left(n_{i}, 2^{Q}\left(n_{i}\right), m\right)\right|_{m=1}-\left.E\left(n_{i}, 2^{Q^{\prime}}\left(\widehat{n}_{i}\right), m\right)\right|_{m=1}}{\left.E\left(n_{i}, 2^{Q}\left(n_{i}\right), m\right)\right|_{m=1}}\right|
$$

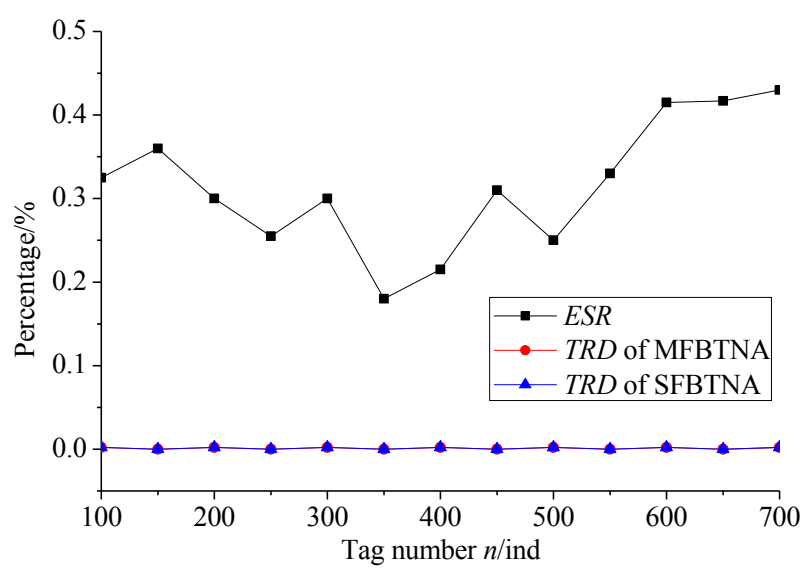

Fig. 6. Curve of estimation and throughput errors of algorithms

According to Eqs.11 and 12, the error curves of ESB and TRD in SFBTNA and the algorithm in this paper are obtained. The number of selected tags is 100-700. As can be seen from the figure, ESR $<0.45 \%$ on the whole, while the TRDs of the two algorithms are basically 0 because each value $\mathrm{Q}$ corresponds to a wider range of tag values, resulting in a minor estimated error, which indicates ESR and TRD basically have no effect on the two algorithms. 


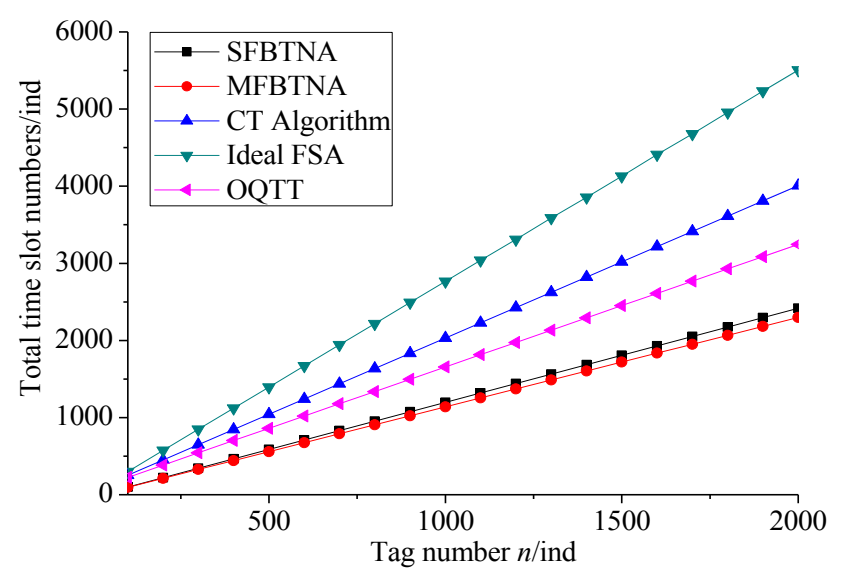

Fig. 7. Comparison curve of overall timeslots of different algorithms

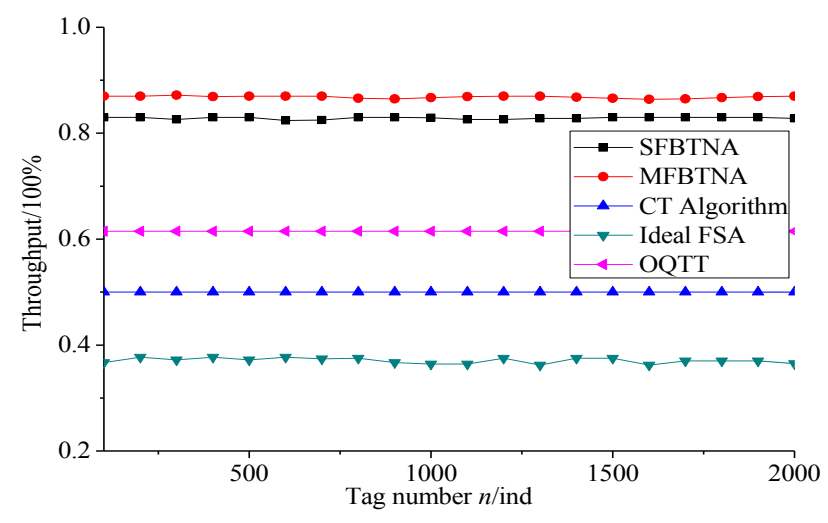

Fig. 8. Comparison and analysis of throughput rates of different algorithms

As shown in Fig. 7, the total timeslots of different algorithms are compared. It can be seen from the figure that the total number of timeslots of FSA is maximum since the FSA is built based on the ALOHA and generates the free time slots during the information transmission. There is no better anti-collision mechanism in the algorithm; though there is no free timeslot in CT, the information collision is more frequent. The total number of timeslots of CT is only less than that of FSA, but the figure is still more on the whole; the algorithm proposed in this paper uses the probe detection to eliminate the free timeslots. CT algorithm reduces the collision probability of the label transmission information, so the total number of the timeslots is minimized.

The curves of the throughput rates of the five algorithms are shown in Fig. 8. The throughput of the algorithm proposed in this paper reaches $87 \%$, and that of SFBTNA is $82.7 \%$. The estimation error has little impact on the above two algorithms. In contrast, OQTT's throughput rate is $61 \%$, the throughput rates of CT and FSA are $49 \%$ and $37 \%$. 
The average time-consuming comparison of the five algorithms is shown in Fig. 9. As can be seen from the figure, the time spent in the computation is in turn CT, FSA, OQTT, SFBTNA and the algorithm proposed in this paper. The availability and superiority of the proposed algorithm.

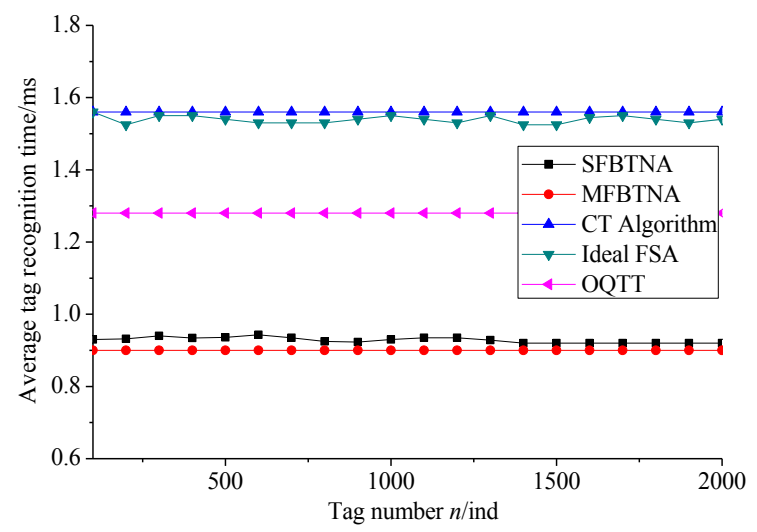

Fig. 9. Comparison and analysis of tag evaluation recognition for different algorithms

\section{Conclusion}

Based on the traditional anti-collision algorithm for RFID system, an improved adaptive $\mathrm{N}$-tree collision algorithm was constructed by incorporation of maximum likelihood estimation and probe pre-detection. The proposed algorithm integrates the Aloha- and tree-based features of anti-collision algorithms, and effectively restrains the starvations of the two algorithms, fill the gap that the label collision probability is higher. The conclusions are drawn as follows:

(1) The proposed improved adaptive N-tree anti-collision algorithm can adaptively choose the value $\mathrm{N}$ of the tree and reduce the length of the free timeslot, eliminate the defects of the traditional tree-based algorithm whose tags are classified more and the collision probability is higher. The level-by-level frame identification is used to build $\mathrm{N}$-tree to eliminate the free timeslot and improve the tag identification precision of the RFID system.[26]

(2) The results from simulation experiments show that the proposed algorithm has minor ESR and TRD and features large throughput rate (87\%), low label recognition delay and minimum timeslots, hence to be well applied in a large-scale logistics and other fields with fast information recognition involved.

\section{Acknowledgment}

Research on Construction and Application of Seafarer Certificate System Based on Marine-Characterized Resource Platform(A modern educational technology research project of Jiangsu academy of education and Science: 2016-R-49979) 


\section{$7 \quad$ References}

[1] Ullah, S., Alsalih, W., Alsehaim, A., Alsadhan, N. (2012). A review of tags anti-collision and localization protocols in RFID networks. Journal of Medical Systems, 36(6): 40374050. HTTPS://DOI.ORG/ 10.1007/s10916-012-9876-5

[2] Min-Bo, L. I., Jin, Z. X., Chen, C. (2010). Application of RFID on products tracking and tracing system. Computer Integrated Manufacturing Systems, 16(1): 202-208.

[3] Wu, Y., Sheng, Q. Z., Shen, H., Zeadally, S. (2013). Modeling object flows from distributed and federated RFID data streams for efficient tracking and tracing. IEEE Transactions on Parallel \& Distributed Systems, 24(10): 2036-2045. HTTPS://DOI.ORG/10.1 109/tpds.2013.99

[4] Choi, S., Choi, J., Yoo, J. (2013). Mrct: an efficient tag identification protocol in RFID systems with capture effect. Ksii Transactions on Internet \& Information Systems, 7(7): 1624-1637. HTTPS://DOI.ORG/10.3837/tiis.2013.07.006

[5] Liang, C. K., Chien, Y. C., Tsai, C. H. (2013). A pre-detection query tree tag anti-collision scheme in RFID systems. 51-56.

[6] Alsalih, W., Ali, K., Hassanein, H. (2013). A power control technique for anti-collision schemes in RFID systems. Computer Networks, 57(9): 1991-2003. HTTPS://DOI.ORG/10.1016/j.comnet.2013.03.016

[7] Shih, D. H., Sun, P. L., Yen, D. C., Huang, S. M. (2006). Taxonomy and survey of RFID anti-collision protocols. Computer Communications, 29(11): 2150-2166. HTTPS://DOI.ORG/10.1016/j.comcom.2005.12.011

[8] Klair, D. K., Chin, K. W., Raad, R. (2010). A survey and tutorial of RFID anti-collision protocols. IEEE Communications Surveys \& Tutorials, 12(3): 400-421. HTTPS://DOI.ORG/10.1109/surv.2010.031810.00037

[9] Joo, Y. I., Seo, D. H., Kim, J. W. (2014). An efficient anti-collision protocol for fast identification of RFID tags. Wireless Personal Communications, 77(1): 767-775. HTTPS://DOI.ORG/10.1007/s11277-013-1535-8

[10] Dhakal, S., Shin, S. (2014). Precise-optimal frame length based collision reduction schemes for frame slotted aloha RFID systems. Ksii Transactions on Internet \& Information Systems, 8(1): 165-182. HTTPS://DOI.ORG/10.3837/tiis.2014.01.010

[11] Vogt, H. (2002). Efficient object identification with passive RFID tags. Springer Berlin Heidelberg, 3(3): 98-113. HTTPS://DOI.ORG/10.1007/3-540-45866-2_9

[12] Hu, M. S., Lv, G. N. (2011). Anti-collision algorithm for RFID based on dynamic packet query tree. Advanced Materials Research, 159: 550-555. HTTPS://DOI.ORG/10.4028/www.scientific.net/amr.159.550

[13] Li, X., Feng, Q. (2013). Grouping based dynamic framed slotted aloha for tag anticollision protocol in the mobile RFID systems. Applied Mathematics \& Information Sciences, 7(2L): 655-660. HTTPS://DOI.ORG/10.12785/amis/072140

[14] Pan, W., Xie, Q., Li, S., Yang, G., Feng, N. (2012). Anti-collision algorithm based on dynamic grouping mechanism in RFID systems. Journal of Convergence Information Technology, 7(15): 338-346. HTTPS://DOI.ORG/10.4156/jcit.vol7.issue15.40

[15] Cmiljanic, N., Landaluce, H., Perallos, A., Arjona, L. (2017). Influence of the distribution of tag ids on RFID memoryless anti-collision protocols. Sensors, 17(8): 1891. HTTPS://DOI.ORG/10.3390/s17081891

[16] Shih, D. H., Sun, P. L., Yen, D. C., Huang, S. M. (2006). Taxonomy and survey of RFID anti-collision protocols. Computer Communications, 29(11): 2150-2166. HTTPS://DOI.ORG/10.1016/j.comcom.2005.12.011 
[17] Choi, S. H., Yang, B., Cheung, H. H., Yang, Y. X. (2015). RFID tag data processing in manufacturing for track-and-trace anti-counterfeiting. Computers in Industry, 68: 148-161. HTTPS://DOI.ORG/10.1016/j.compind.2015.01.004

[18] Yeh, M. K., Jiang, J. R. (2009). Adaptive splitting and pre-signaling for RFID tag anticollision. Computer Communications, 32(17): 1862-1870. HTTPS://DOI.ORG/10.1016/j. comcom.2009.07.011

[19] Yeo, H. J., Yong, H. K., Lim, H. Y., Yong, S. P., Ahn, K. S. (2007). ID prediction algorithm for tag collision arbitration in RFID system. IEEE International Conference on Embedded and Real-Time Computing Systems and Applications, 53(4): 476-481. HTTPS://DOI.ORG/10.1109/rtcsa.2007.43

[20] Myung, J., Lee, W. (2006). Adaptive binary splitting: a RFID tag collision arbitration protocol for tag identification. Mobile Networks \& Applications, 11(5): 711-722. HTTPS://DOI.ORG/10.1109/icbn.2005.1589637

[21] Zheng, F., Kaiser, T. (2016). Adaptive aloha anti-collision algorithms for RFID systems. Eurasip Journal on Embedded Systems, 2016(1): 7. HTTPS://DOI.ORG/10.1186/s13639016-0029-7

[22] Klair, D., Chin, K. W., Raad, R. (2009). On the energy consumption of pure and slotted aloha based RFID anti-collision protocols. Computer Communications, 32(5): 961-973. HTTPS://DOI.ORG/10.1016/j.comcom.2008.12.019

[23] Joshi, G. P., Kim, S. W. (2008). Survey, nomenclature and comparison of reader anticollision protocols in RFID. Iete Technical Review, 25(5): 234-243. HTTPS://DOI.ORG/10.4103/0256-4602.44659

[24] Deng, D. J., Tsao, H. W. (2011). Optimal dynamic framed slotted aloha based anticollision algorithm for RFID systems. Wireless Personal Communications, 59(1): 109-122. HTTPS://DOI.ORG/10.1007/s11277-010-0193-3

[25] Wang, Y. P., Sheng, H., Wang, X. L., Zhang, X. B. (2013). Anti-collision algorithm analysis of aloha-based RFID. Applied Mechanics \& Materials, 273(1): 745-749. HTTPS://DOI.ORG/10.4028/www.scientific.net/amm.273.745

[26] Das, A., Deb, K., Bajerjee, S., Bag, R. (2017). A new method for tutorial gap identification towards students modeling, Mathematical Modelling of Engineering Problems, 4(2): 8083. HTTPS://DOI.ORG/ 10.18280/mmep.040203

\section{Authors}

Yuxia Li worked in Hebei agricultural university network center, the Jiangsu maritime college library technician, intelligence, deputy director of the institute, Sichuan university, master of software engineering, electronic information engineers, part-time teachers school curricula, any information IT community guide teacher

Zhinan Zhou now work in modern education technology centre of Hebei Agricultural University, Research Associate, research direction computer science and technology, Master of Engineering in Agricultural Mechanization Engineering, Hebei Agricultural University.

Article submitted 15 July 2018. Resubmitted 28 July 2018. Final acceptance 16 September 2018. Final version published as submitted by the authors. 